\title{
SIX1 is overexpressed in endometrial carcinoma and promotes the malignant behavior of cancer cells through ERK and AKT signaling
}

\author{
XIAOCHUAN XIN, YUE LI and XIANGHONG YANG \\ Department of Pathology, Shengjing Hospital of China Medical University, Shenyang, Liaoning 110004, P.R. China
}

Received April 9, 2015; Accepted July 7, 2016

DOI: $10.3892 / \mathrm{ol} .2016 .5098$

\begin{abstract}
The sineoculis homeobox homolog 1 (SIX1) protein has been found to be important for cancer progression. However, its biological role in human endometrial carcinomas remains unexplored. The potential mechanism of SIX1-induced cancer progression remains unclear. In the present study, SIX1 protein expression was examined in 84 cases of endometrial carcinoma tissues using immunohistochemisty, and SIX1 was found to be overexpressed in $51.1 \%$ (43/84) of cervical cancer cells. Small interfering RNA (siRNA) knockdown of SIX1 was also performed in Ishikawa cells with high endogenous SIX1 expression, and SIX1 was overexpressed in the HEC1B cell line with low endogenous expression. SIX1 overexpression promoted cell grow th rate and colony formation ability, whereas SIX1 depletion inhibited cell growth and colony formation. Further analysis showed that SIX1 knockdown downregulated, and SIX1 overexpression upregulated, cyclin D1, cyclin E, phosphorylated (p-)extracellular signal-regulated kinase (ERK), and p-protein kinase B (AKT) expression. The ERK inhibitor, U0126, and AKT inhibitor treatments blocked the effect of SIX1 on proliferation. In conclusion, the present study found that SIX1 overexpression promotes cancer cell growth in endometrial carcinoma, possibly through ERK- and AKT-mediated pathways.
\end{abstract}

\section{Introduction}

Endometrial carcinoma is the most common gynecological malignancy, and the incidence is rising (1). Patients are standardly surgically treated with a hysterectomy and bilateral salpingoophorectomy. Despite the improved surgical treatment and adjuvant therapy used in previous studies, the prognosis of

Correspondence to: Professor Xianghong Yang, Department of Pathology, Shengjing Hospital of China Medical University, 36 Sanhao Street, Shenyang, Liaoning 110004, P.R. China E-mail: yangxianghong_sj@126.com

Key words: sineoculis homeobox homolog 1, proliferation, endometrial carcinoma, extracellular signal-regulated kinase, protein kinase B endometrial carcinoma has not improved significantly (2-5). Identifying novel markers that can be used to predict the risk of endometrial carcinoma progression remains to be important.

Sineoculis homeobox homolog 1 (SIX1) is a transcription factor that belongs to the SIX family of homeoproteins. SIX1 expression level is increased in embryogenesis and promotes progenitor cell expansion and survival (6-8). SIX1 absence results in the reduction in size or loss of numerous organs, as a result of inhibited proliferation and increased apoptosis in mice (9). However, SIX1 expression is low in adult tissues, and aberrant expression of the SIX1 gene in adult tissue may contribute to carcinogenesis (10). SIX1 was found to be overexpressed in human breast, cervical, ovarian and pancreatic cancers and associated with a poor patient survival (11-15). SIX1 overexpression promotes cancer cell survival and epithelial to mesenchymal transition (EMT) $(16,17)$. Overall, these findings suggest that SIX1 is an important oncoprotein for the development of cancer. However, the clinical significance and biological role of SIX1 in endometrial carcinoma remains unexplored. In the present study, endogenous SIX1 expression was examined in endometrial carcinoma specimens. SIX1 expression was overexpressed and downregulated, and the effect on cell proliferation was investigated. The present study also investigated the potential molecular signaling pathways underlying the biological effects of SIX1.

\section{Materials and methods}

Patients and specimens. The study protocol was approved by the Institutional Review Board of Shengjing Hospital of China Medical University (Shengyang, China). Primary tumor specimens were obtained from 60 female patients (age range, 42-70 years) diagnosed with endometrioid adenocarcinoma, who underwent resections in the Shengjing Hospital of China Medical University between January 2010 and December 2012. The tumor sections were evaluated using histological diagnosis, according to World Health Organization guidelines (18-20). The International Federation of Gynecology and Obstetrics (FIGO) staging system was used to classify patients as stages I, II or III (21). Clinical and histopathological data were obtained from medical records. The study protocol was approved by the Institutional Review Board of Shengjing Hospital of China Medical University (Shenyang, China) and informed consent was obtained from all patients. 
Immunohistochemistry. Tumor specimens were fixed with $10 \%$ neutral formalin and $4-\mu \mathrm{m}$ thick paraffin sections were cut. Immunostaining was performed using the Ultrasensitive $^{\text {TM }}$ S-P staining kit (Maixin Biotech Co., Ltd., Fuzhou, China). After antigen retrieval in citrate buffer $(\mathrm{pH}$ 6.0) for $2 \mathrm{~min}$ in an autoclave, $0.3 \%$ hydrogen peroxide was applied to the samples for $15 \mathrm{~min}$ and then the sections were incubated with goat serum for $10 \mathrm{~min}$ at room temperature (Invitrogen; Thermo Fisher Scientific, Inc., Waltham, MA, USA). The samples were incubated with SIX1 rabbit polyclonal antibody (dilution, 1:300; SAB2102157; Sigma-Aldrich; Merck Millipore, Darmstadt, Germany) at $4^{\circ} \mathrm{C}$ overnight. Samples were then incubated with biotinylated goat anti-rabbit serum immunoglobulin G (IgG) antibodies (dilution, 1:200; A0545; Sigma-Aldrich; Merck Millipore) for $10 \mathrm{~min}$ at room temperature subsequent to washing in phosphate-buffered saline (PBS). The samples were then incubated with streptavidinbiotin antibodies conjugated with horseradish peroxidase (dilution, 1:300; A0185; Sigma-Aldrich; Merck Millipore) for $10 \mathrm{~min}$ at room temperature. The DAB Detection kit (Maixin Biotech Co., Ltd.) was then used for staining. Counterstaining with hematoxylin was performed, and the sections were dehydrated in ethanol prior to mounting.

Two independent pathologists of the Department of Pathology of Shengjing Hospital of China Medical University examined the sections. In total, 500 cells were counted for each slide. The immunostaining of SIX1 was scored on a system that evaluated the intensity and percentage of tumor cells. Nuclear and cytoplasmic immunostaining in tumor cells were considered to show SIX1 staining. The intensity of staining was scored as: 0 , no signal; 1 , weak; or 2, strong. Percentage scores were assigned as: $1,1-25 \% ; 2,26-50 \% ; 3,51-75 \%$; or $4,76-100 \%$. Then the intensity score and percentage score were multiplied to get a final score between 0 and 8 . Tumor samples receiving a score between 4 and 8 were regarded to show SIX1 overexpression.

Cell culture and transfection. The HEC1B and Ishikawa cell lines were obtained from American Type Culture Collection (Manassas, VA, USA). Cells were cultured in Dulbecco's modified Eagle's medium (DMEM; Invitrogen; Thermo Fisher Scientific, Inc.) containing 10\% fetal calf serum (Invitrogen; Thermo Fisher Scientific, Inc.). Cells were passaged every 2 days with $0.25 \%$ trypsin.

The pCMV6-SIX1 vector was purchased from OriGene Technologies, Inc. (Rockville, MD, USA). Attractene transfection reagent (Qiagen, Hilden, Germany) was used for plasmid transfection. The pCMV6 vector was used as a negative control. Cells were harvested $48 \mathrm{~h}$ subsequent to transfection. Dharmacon On-TargetPlus SMARTpool small interfering RNA (siRNA) for SIX1 and ON-TARGETplus Non-targeting siRNA were purchased from Thermo Fisher Scientific, Inc. The cells were transfected with siRNA using the DharmaFECT 1 (Thermo Fisher Scientific, Inc.), according to the manufacturer's protocol.

Reverse transcription-quantitative polymerase chain reaction (RT-qPCR) (SYBR Green method). Total RNA was extracted using a total RNA extraction kit (SYBR Select Master Mix; Applied Biosystems; Thermo Fisher Scientific, Inc.). RT-qPCR was performed using the Applied Biosystems SYBR Green master mix kit, purchased from Thermo Fisher Scientific, Inc. PCR was performed using 7900HT Real-Time PCR System (Applied Biosystems; Thermo Fisher Scientific, Inc.). $\beta$-actin was used as the reference gene. The cycling conditions were as follows: $50^{\circ} \mathrm{C}$ for $2 \mathrm{~min}, 95^{\circ} \mathrm{C}$ for $2 \mathrm{~min}$, and 45 cycles of $95^{\circ} \mathrm{C}$ for $15 \mathrm{sec}$ and $60^{\circ} \mathrm{C}$ for $40 \mathrm{sec}$. The relative expression of target genes were calculated as $\Delta \mathrm{Cq}=\mathrm{Cq}$ gene $-\mathrm{Cq}$ reference, and the fold change of target gene expression was calculated by the $2^{-\Delta \Delta \mathrm{Cq}}$ method (22). Experiments were repeated in triplicate. The primer sequences were as follows: SIX1 forward, 5'-AAG GAGAAGTCGAGGGGTGT-3' and reverse, 5'-TGCTTGTTG GAGGAGGAGTT-3'; $\beta$-actin forward, 5'-ATAGCACAGCCT GGATAGCAACGTAC-3' and reverse, 5'-CACCTTCTACAA TGAGCTGCGTGTG-3'.

Western blot analysis. Total proteins from the cells were extracted using lysis buffer and quantified using the Bradford method (23-25). In total, $30 \mu \mathrm{g}$ protein was separated by sodium dodecyl sulfate polyacrylamide gel electrophoresis (stacking gel, 5\%; separating gel, 10\%). Samples were transferred to polyvinylidene fluoride (PVDF) membranes (EMD Millipore, Billerica, MA, USA) and subjected to a blocking step with 5\% bovine serum albumin (BSA) (5 g BSA + $100 \mathrm{ml}$ Tris-buffered saline with Tween 20; Maixin Biotech Co., Ltd.) at room temperature for $45 \mathrm{~min}$. Next, membranes were incubated overnight at $4^{\circ} \mathrm{C}$ with antibodies against SIX1 (rabbit polyclonal antibody; dilution, 1:800; SAB2102157; Sigma-Aldrich; Merck Millipore), phosphorylated (p)-retinoblastoma protein (Rb) (rabbit monoclonal antibody; dilution, 1:1,000; 8147; Cell Signaling Technology, Inc., Danvers, MA, USA), p-ERK (rabbit polyclonal antibody; dilution, 1:1,000; 9101; Cell Signaling Technology, Inc.), ERK (rabbit polyclonal antibody; dilution, 1:1,000; 9102; Cell Signaling Technology, Inc.), p-AKT (rabbit polyclonal antibody; dilution, 1:1,000; 9271; Cell Signaling Technology, Inc.), AKT (rabbit polyclonal antibody; dilution, 1:1,000; 9272; Cell Signaling Technology, Inc.), cyclin D1 (rabbit monoclonal antibody; dilution, 1:1,000; 2978; Cell Signaling Technology, Inc.), cyclin E (rabbit monoclonal antibody; dilution, 1:1,000; 20808; Cell Signaling Technology,Inc.) and glyceraldehyde 3-phosphate dehydrogenase (GAPDH; rabbit polyclonal antibody; dilution, 1:1,000; G5262; Santa Cruz Biotechnology, Inc., Dallas, TX, USA). Subsequently, samples were incubated with peroxidasecoupled anti-mouse (monoclonal antibody; dilution, 1:1,000; 7076, Cell Signaling Technology, Inc.) or anti-rabbit IgG antibodies (polyclonal antibody; dilution, 1:1,000; 7074; Cell Signaling Technology, Inc.) at $37^{\circ} \mathrm{C}$ for $2 \mathrm{~h}$. Target proteins on the PVDF membrane were visualized using a Pierce enhanced chemiluminescence kit (Thermo Fisher Scientific, Inc.) and captured using a DNR BioImaging system equipped with GelCapture application software (DNR Bio-Imaging Systems Ltd., Jerusalem, Israel).

Colony formation and 3-(4,5-dimethylthiazol-2-yl)-2,5-diphenyltetrazolium bromide (MTT) assays. For the colony formation assay, cells $(\sim 1,000$ per dish) were plated into $6 \mathrm{~cm}$ culture dishes, $48 \mathrm{~h}$ after transfection. After two weeks, plates were washed with PBS and Giemsa staining was performed to visualize the colonies. The colonies with $>50$ cells were counted using a microscope. 
Table I. Distribution of SIX1 status in endometrial carcinomas, according to clinicopathological characteristics.

\begin{tabular}{|c|c|c|c|c|}
\hline Characteristics & No. of patients & SIX1 weak/negative & SIX1 positive & P-value \\
\hline Age, years & & & & 0.6089 \\
\hline$<60$ & 53 & 27 & 26 & \\
\hline$\geq 60$ & 31 & 14 & 17 & \\
\hline Tumor grade & & & & 0.0105 \\
\hline $1+2$ & 61 & 35 & 26 & \\
\hline 3 & 23 & 6 & 17 & \\
\hline FIGO stage & & & & 0.7034 \\
\hline I & 59 & 28 & 31 & \\
\hline II+III & 25 & 13 & 12 & \\
\hline
\end{tabular}

SIX1, sineoculis homeobox homolog 1; FIGO, International Federation of Gynecology and Obstetrics.
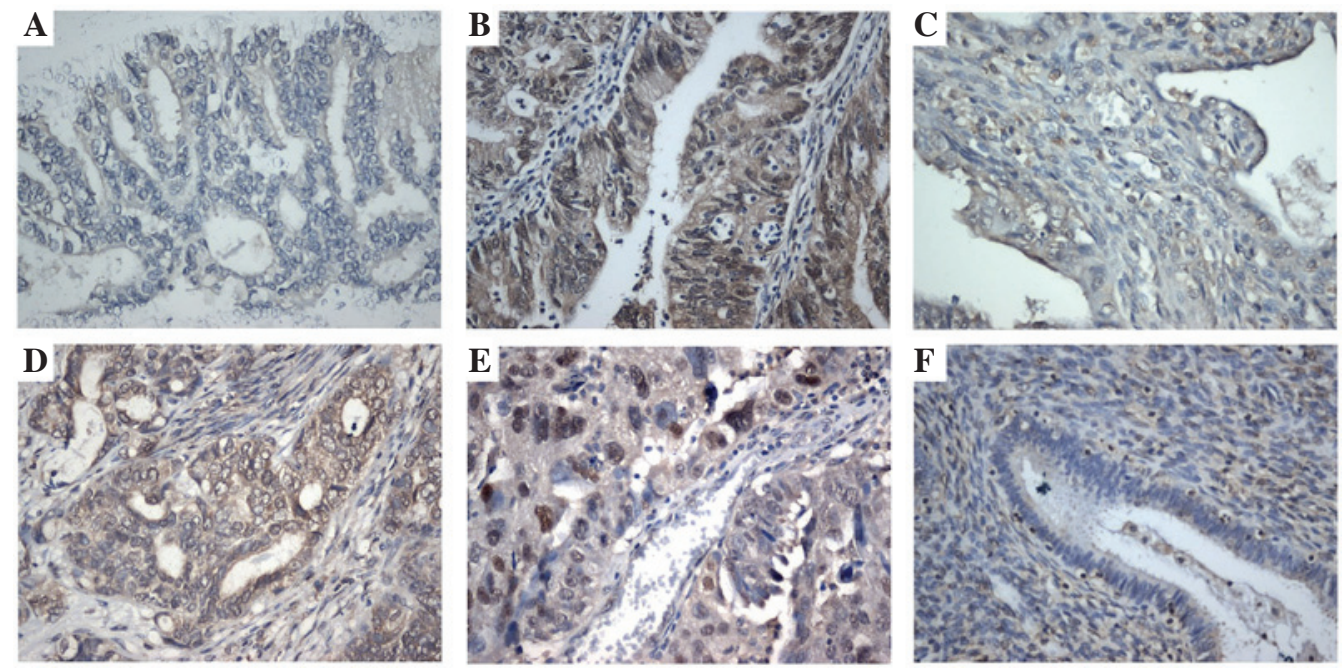

Figure 1. Expression of SIX1 protein in endometrial carcinoma tissues. (A) Negative and (B) positive SIX1 expression in grade 1 endometrial carcinoma. (C) Negative and (D) positive expression of SIX1 in a case of grade 2 endometrial carcinoma. (E) Positive SIX1 expression in a case of grade 3 endometrial carcinoma. (F) Negative expression in normal endometrial tissues. Magnification, x400. SIX1, sineoculis homeobox homolog 1.

B

A
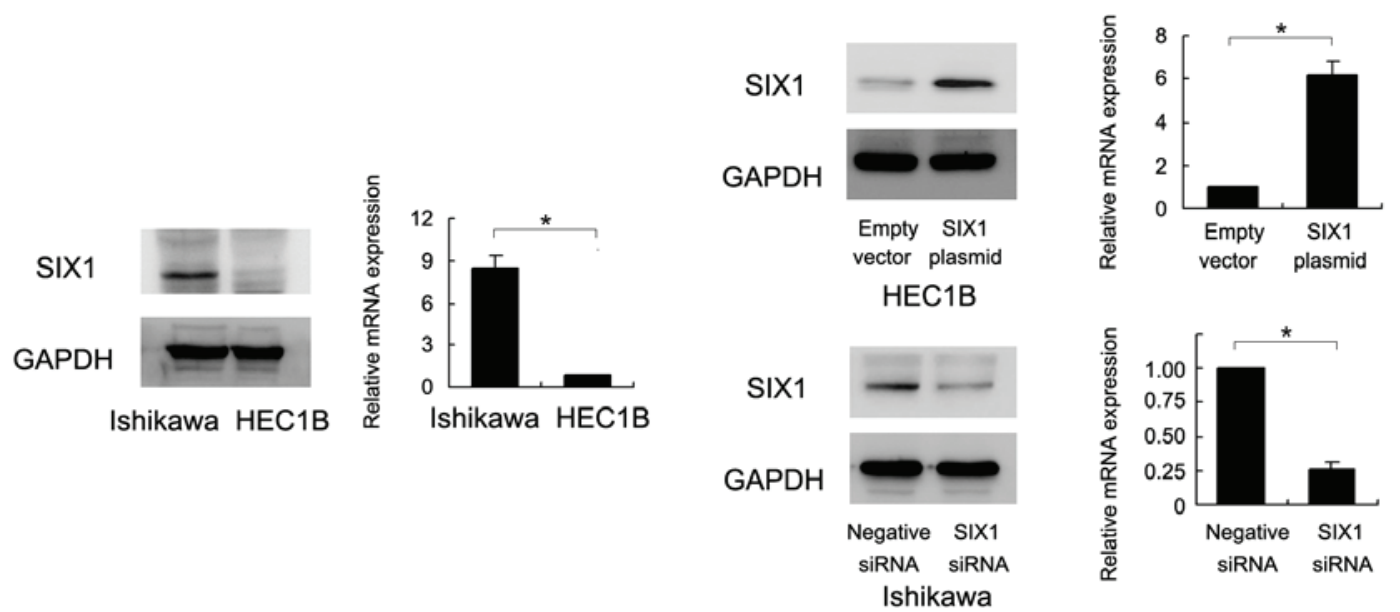

Figure 2. Transfection efficiency of SIX1 plasmid and siRNA in HEC1B and Ishikawa endometrial carcinoma cell lines. (A) Western blot analysis and RT-qPCR showed that SIX1 mRNA expression was increased in Ishikawa cells and decreased in HEC1B cells. "P<0.05, empty vector vs. SIX1 plasmid. (B) Western blot analysis and RT-qPCR showed that SIX1 transfection in the HEC1B cell line upregulated SIX1 protein and mRNA expression. SIX1 siRNA treatment in the Ishikawa cell line resulted in downregulated SIX1 protein and mRNA expression. "P<0.05, negative siRNA vs. SIX1 siRNA. SIX1, sineoculis homeobox homolog 1; siRNA, small interfering RNA; RT-qPCR, reverse transcription-quantitative polymerase chain reaction; mRNA, messenger RNA. 
A
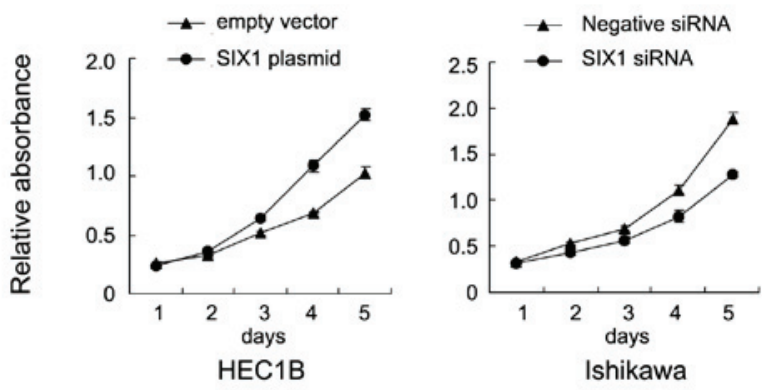

B

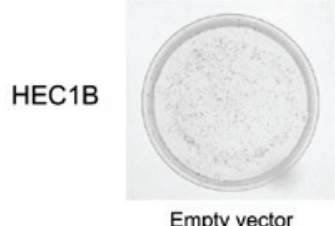

Empty vector

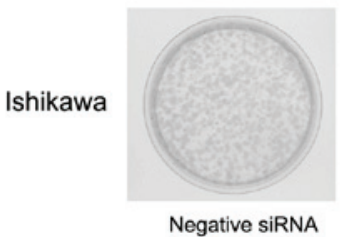

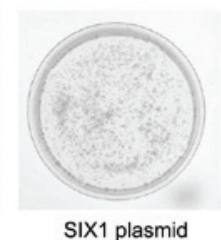

SIX1 plasmid

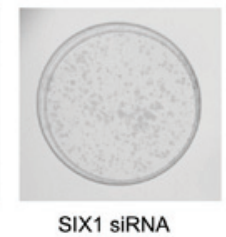

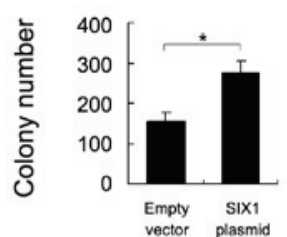

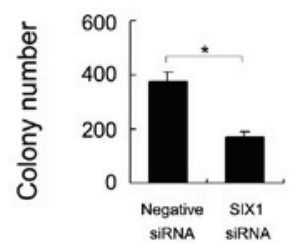

Figure 3. SIX1 transfection promotes proliferation and colony formation. (A) 3-(4,5-dimethylthiazol-2-yl)-2,5-diphenyltetrazolium bromide assay showed that SIX1 transfection upregulated cell proliferation rate in HEC1B cells, and SIX1 siRNA downregulated cell proliferation rate in Ishikawa cells. (B) Colony formation assay showed that SIX1 transfection increased ("P $<0.05$, empty vector vs. SIX1 plasmid), while SIX1 depletion decreased ("P $<0.05$, negative siRNA vs. SIX1 siRNA), colony formation ability. SIX1, sineoculis homeobox homolog 1; siRNA, small interfering RNA.

A

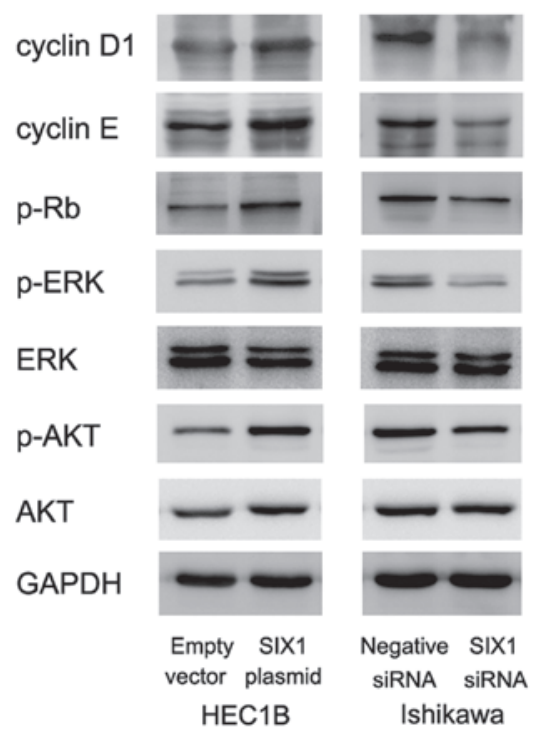

B

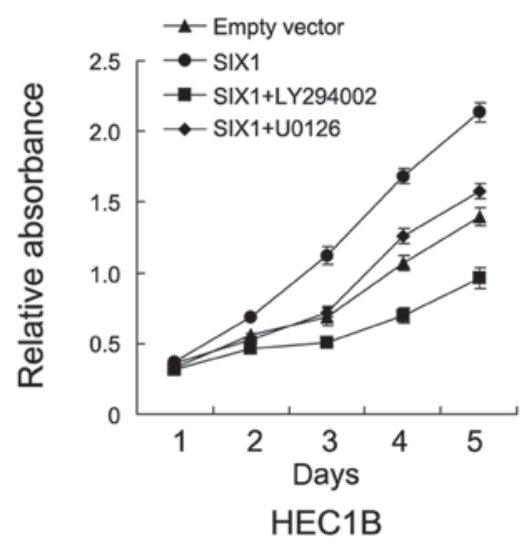

Figure 4. SIX1 upregulates ERK and AKT activity. (A) SIX1 transfection was performed in HEC1B cells and siRNA knockdown was performed in Ishikawa cells. Western blot analysis showed that SIX1 transfection upregulated, while SIX1 depletion downregulated, cyclin D1, cyclin E, p-ERK and p-AKT expression. (B) In SIX1 transfected HEC1B cells, treatment with AKT inhibitor, LY294002 (20 $\mu \mathrm{M})$, and ERK inhibitor, U0126 (10 $\mu$ mol/l), significantly blocked the role of SIX1 on cell growth rate. SIX1, sineoculis homeobox homolog 1; siRNA, small interfering RNA; ERK, extracellular signal-regulated kinase; AKT, protein kinase B; p-ERK, phosphorylated-ERK; p-AKT, phosphorylated-AKT; p-Rb, phosphorylated-retinoblastoma protein.

For the MTT assay, cells $(\sim 3,000$ per well) were plated in 96-well plates and cultured for 5 days. In total, $20 \mu 1$ of $5 \mathrm{mg} / \mathrm{ml}$ MTT solution was added to each well. After incubation for $4 \mathrm{~h}$, the medium was removed and the remaining MTT formazan was dissolved in $150 \mu \mathrm{l}$ of DMSO. The optical density of the solution was measured at $490 \mathrm{~nm}$ using a microplate reader. 
Statistical analysis. SPSS version 11.5 for Windows (SPSS, Inc., Chicago, IL, USA) was used for all statistical analyses. A $\chi^{2}$ test was used to examine the possible associations between SIX1 expression and clinicopathological factors. The Student's $t$-test was used to compare differences between control and SIX1-transfected cells. All P-values were based on a two-sided statistical analysis, and $\mathrm{P}<0.05$ was considered to indicate a statistically significant difference.

\section{Results}

Expression of SIX1 in human endometrial carcinoma. SIX1 immunostaining was observed in 84 cases of endometrioid adenocarcinoma (Fig. 1). In normal endometrial tissues, stromal and glandular tissues showed weak or negative staining. In total, 43/84 cancer tissues showed positive SIX1 immunoreactivity, and the expression was located in the nuclear and cytoplasmic compartment of the cancer cells. The associations between SIX1 expression and the clinicopathological characteristics of patients is shown in Table I. A high SIX1 immunostaining score in the primary endometrioid carcinoma was significantly associated with advanced tumor grade $(\mathrm{P}=0.0105)$. However, there was no association between SIX1 expression levels and other parameters, including FIGO stage $(\mathrm{P}=0.7034)$ or patient age $(\mathrm{P}=0.6089)$.

SIX1 promotes endometrial cell proliferation and colony formation. The endogenous expression of SIX1 was examined by western blot analysis and RT-qPCR in Ishikawa and HEC1B cell lines. Ishikawa cells possess high endogenous SIX1 expression and HEC1B cells possess low SIX1 expression (Fig. 2A). To determine the biological role of SIX1 in endometrial cancer, plasmid transfection and siRNA knockdown were performed. As shown in Fig. 2B, SIX1 plasmid transfection significantly upregulated the expression of SIX1 protein and mRNA. SIX1 siRNA transfection significantly downregulated the expression of SIX1 protein and mRNA. The MTT assay revealed that SIX1 upregulation increased the cell proliferation rate in HEC1B cells and SIX1 depletion decreased the proliferation rate in Ishikawa cells (Fig. 3A). A colony formation assay was also performed. As shown in Fig. 3B, SIX1 transfection significant increased the colony number of HEC1B cells (empty vector vs. SIX1 plasmid, $155 \pm 22$ vs. $276 \pm 28 ; \mathrm{P}=0.041)$. By contrast, SIX1 depletion in Ishikawa cells significantly downregulated colony formation ability (negative siRNA vs. SIX1 siRNA, $378 \pm 33$ vs. $169 \pm 20$; $\mathrm{P}=0.047$ ). Accordingly, SIX1 transfection was found to upregulate, whereas SIX1 depletion was found to downregulate, cyclin D1 and cyclin E and p-Rb expression (Fig. 4), which suggests that SIX1 facilitated cell cycle transition.

SIX1 promotes cell proliferation through ERK and AKT signaling. In order to investigate the potential mechanisms of SIX1-induced endometrial carcinoma cell proliferation, the level of ERK and AKT signaling pathway activation was examined in cells with SIX1 transfection and depletion. SIX1 transfection was found to upregulate AKT and ERK phosphorylation, whereas SIX1 depletion was found to decrease the level of pathway activation (Fig. 4A). In addition, treatment with the AKT inhibitor, LY294002 $(20 \mu \mathrm{M})$, and ERK inhibitor, U0126
(10 $\mu \mathrm{mol} / \mathrm{l})$, for $6 \mathrm{~h}$ significantly blocked the role of SIX1 on endometrial cancer growth (Fig. 4B), suggesting that the ERK and AKT signaling cascades were involved in SIX1-induced endometrial cancer growth.

\section{Discussion}

Endometrial carcinoma is a common gynecological cancer. Although studies have indicated that numerous oncogenes in endometrial carcinoma promote cancer progression, novel markers to predict aggressive phenotypes are urgently required. The upregulation of SIX1 expression has been reported in human gynecological cancers, including breast, cervical and ovarian carcinoma, where it leads to increased proliferation and metastasis $(13-15,17)$. One study reported that SIX1 was aberrantly upregulated in human colorectal cancer and promoted epithelial-mesenchymal transition via zinc finger E-box binding homeobox 1 activation (26). It was recently reported that the SIX1 protein is upregulated in human gastric carcinoma and associated with clinical parameters (27). However, the biological roles and potential molecular mechanism of SIX1 in human endometrial carcinoma cells remains unexplored.

The present study found that SIX1 was overexpressed in 43/84 endometrial carcinoma samples and associated with advanced tumor grade. These findings indicate an association between SIX1 overexpression and the malignant endometrial carcinoma phenotype. In addition, SIX1 overexpression promoted cell proliferation, while SIX1 depletion inhibited cell growth. These data are in accordance with previous reports, which confirmed the role of SIX1 as an important oncogene in human endometrial carcinomas $(15,26,28,29)$.

The regulation effect of SIX1 on cell cycle proteins has been previously reported in other human cancers $(30,31)$. The present study examined the effect of SIX1 on cell cycle progression and found that SIX1 overexpression upregulated, while SIX1 depletion downregulated, cyclin D1 and cyclin E expression; a finding that was in accordance with previous reports $(15,32,33)$. The present study further investigated the molecular pathways involved in SIX1-induced cancer progression. Previous reports indicated that SIX1 could induce EMT, which is closely associated with cancer cell invasion and metastasis $(16,17)$. Previous studies indicated that SIX1 could induce ERK activation (34). The present study investigated the change of ERK status in cells transfected with SIX1 plasmids/siRNA. The results showed that SIX1 could increase the level of ERK phosphorylation. The present study also examined the change of AKT phosphorylation in endometrial carcinoma cells with the overexpression or knockdown of SIX1. SIX1 overexpression or knockdown resulted in the increased or decreased Ser473 phosphorylation of AKT, respectively. In addition, the ERK inhibitor, U0126, and AKT inhibitor, LY294002, could partially reverse the upregulatory effect of SIX1 on proliferation, which strongly supports the importance of SIX1 in regulating cell proliferation via the ERK and AKT pathways.

In conclusion, the present study demonstrated that increased SIX1 expression contributes to the malignant tumor growth of endometrial carcinoma cells, possibly through the regulation of the ERK and AKT signaling pathways. SIX1 may be considered as a novel cancer marker for endometrial carcinoma. 


\section{References}

1. Siegel R, Desantis $\mathrm{C}$ and Jemal A: Colorectal cancer statistics, 2014. CA Cancer J Clin 64: 104-117, 2014.

2. Sivridis E, Giatromanolaki A, Gatter KC, Harris AL and Koukourakis MI; Tumor and Angiogenesis Research Group Association of hypoxia-inducible factors 1alpha and 2alpha with activated angiogenic pathways and prognosis in patients with endometrial carcinoma. Cancer 95: 1055-1063, 2002.

3. Sakuragi N, Ohkouchi T, Hareyama H, Ikeda K, Watari H, Fujimoto T, Kuwabara M, Yamamoto R, Sagawa T, Fujino T and Fujimoto $\mathrm{S}$ : Bcl-2 expression and prognosis of patients with endometrial carcinoma. Int J Cancer 79: 153-158, 1998.

4. Kang S, Kang WD, Chung HH, Jeong DH, Seo SS, Lee JM, Lee JK, Kim JW, Kim SM, Park SY and Kim KT: Preoperative identification of a low-risk group for lymph node metastasis in endometrial cancer: A Korean gynecologic oncology group study. J Clin Oncol 30: 1329-1334, 2012.

5. Wright JD, Burke WM, Wilde ET, Lewin SN, Charles AS Kim JH, Goldman N, Neugut AI, Herzog TJ and Hershman DL: Comparative effectiveness of robotic versus laparoscopic hysterectomy for endometrial cancer. J Clin Oncol 30: 783-791, 2012.

6. Liu Y, Chakroun I, Yang D, Horner E, Liang J, Aziz A, Chu A, De Repentigny Y, Dilworth FJ, Kothary R and Blais A: Six 1 regulates MyoD expression in adult muscle progenitor cells PLoS One 8: e67762, 2013.

7. Nord H, Nygard Skalman L and von Hofsten J: Six1 regulates proliferation of Pax7-positive muscle progenitors in zebrafish. J Cell Sci 126: 1868-1880, 2013.

8. Ikeda K, Kageyama R, Suzuki Y and Kawakami K: Six1 is indispensable for production of functional progenitor cells during olfactory epithelial development. Int J Dev Biol 54: 1453-1464, 2010.

9. Chen B, Kim EH and Xu PX: Initiation of olfactory placode development and neurogenesis is blocked in mice lacking both Six1 and Six4. Dev Biol 326: 75-85, 2009.

10. Kumar JP: The sine oculis homeobox (SIX) family of transcription factors as regulators of development and disease. Cel Mol Life Sci 66: 565-583, 2009.

11. Coletta RD, Christensen K, Reichenberger KJ, Lamb J, Micomonaco D, Huang L, Wolf DM, Müller-Tidow C, Golub TR, Kawakami K and Ford HL: The Six1 homeoprotein stimulates tumorigenesis by reactivation of cyclin A1. Proc Natl Acad Sci USA 101: 6478-6483, 2004.

12. Reichenberger KJ, Coletta RD, Schulte AP, Varella-Garcia M and Ford HL: Gene amplification is a mechanism of Sixl overexpression in breast cancer. Cancer Res 65: 2668-2675, 2005.

13. Zheng XH, Liang PH, Guo JX, Zheng YR, Han J, Yu LL Zhou YG and Li L: Expression and clinical implications of homeobox gene Six1 in cervical cancer cell lines and cervica epithelial tissues. Int J Gynecol Cancer 20: 1587-1592, 2010.

14. Behbakht K, Qamar L, Aldridge CS, Coletta RD, Davidson SA, Thorburn A and Ford H: Six1 overexpression in ovarian carcinoma causes resistance to TRAIL-mediated apoptosis and is associated with poor survival. Cancer Res 67: 3036-3042, 2007.

15. Li Z, Tian T, Lv F, Chang Y, Wang X, Zhang L, Li X, Li L, Ma W, $\mathrm{Wu} \mathrm{J}$ and Zhang $\mathrm{M}$ : Six1 promotes proliferation of pancreatic cancer cells via upregulation of cyclin D1 expression. PLoS One 8: e59203, 2013.

16. Smith AL, Iwanaga R, Drasin DJ, Micalizzi DS, Vartuli RL, Tan AC and Ford HL: The miR-106b-25 cluster targets Smad7, activates TGF- $\beta$ signaling and induces EMT and tumor initiating cell characteristics downstream of Six 1 in human breast cancer. Oncogene 31: 5162-5171, 2012.

17. Radisky DC: Defining a role for the homeoprotein Six1 in EMT and mammary tumorigenesis. J Clin Invest 119: 2528-2531, 2009.
18. Song JH, Kim SH, Lee JH, Cho HM, Kim DY, Kim TH, Kim SY, Baek JY, Oh JH, Nam TK, et al: Significance of histologic tumor grade in rectal cancer treated with preoperative chemoradiotherapy followed by curative surgery: A multi-institutional retrospective study. Radiother Oncol 118: 387-392, 2016.

19. Davidson BA, Foote J, Clark LH, Broadwater G, Ehrisman J, Gehrig P, Graybill W, Alvarez Secord A and Havrilesky LJ: Tumor grade and chemotherapy response in endometrioid endometrial cancer. Gynecol Oncol Rep 27: 3-6, 2016.

20. Mittelbronn M, Schittenhelm J, Lemke D, Ritz R, Nägele T, Weller M, Meyermann R and Beschorner R: Low grade ganglioglioma rapidly progressing to a WHO grade IV tumor showing malignant transformation in both astroglial and neuronal cell components. Neuropathology 27: 463-467, 2007.

21. Saida T, Tanaka YO, Matsumoto K, Satoh T, Yoshikawa H and Minami M: Revised FIGO staging system for cancer of the ovary, fallopian tube, and peritoneum: Important implications for radiologists. Jpn J Radiol 34: 117-124, 2016.

22. Livak KJ and Schmittgen TD: Analysis of relative gene expression data using real-time quantitative PCR and the 2(-Delta Delta C(T)) Method. Methods 25: 402-408, 2001.

23. Barbosa H, Slater NK and Marcos JC: Protein quantification in the presence of poly(ethylene glycol) and dextran using the Bradford method. Anal Biochem 395: 108-110, 2009.

24. Carlsson N, Borde A, Wölfel S, Kerman B and Larsson A: Quantification of protein concentration by the Bradford method in the presence of pharmaceutical polymers. Anal Biochem 411: 116-121, 2011.

25. Lu TS, Yiao SY, Lim K, Jensen RV and Hsiao LL: Interpretation of biological and mechanical variations between the Lowry versus Bradford method for protein quantification. N Am J Med Sci 2: 325-328, 2010 .

26. Ono H, Imoto I, Kozaki K, Tsuda H, Matsui T, Kurasawa Y, Muramatsu T, Sugihara K and Inazawa J: SIX1 promotes epithelial-mesenchymal transition in colorectal cancer through ZEB1 activation. Oncogene 31: 4923-4934, 2012.

27. Lv H, Cui A, Sun F, Zhang Y, Li Y, Li L and Lin Z: Sineoculis homeobox homolog 1 protein as an independent biomarker for gastric adenocarcinoma. Exp Mol Pathol 97: 74-80, 2014.

28. Sun SH, Liu D, Deng YT, Zhang XX, Wan DY, Xi BX, Huang W, Chen Q, Li MC, Wang MW, et al: SIX1 coordinates with TGF $\beta$ signals to induce epithelial-mesenchymal transition in cervical cancer. Oncol Lett 12: 1271-1278, 2016.

29. Wang CA, Jedlicka P, Patrick AN, Micalizzi DS, Lemmer KC, Deitsch E, Casás-Selves M, Harrell JC and Ford HL: SIX1 induces lymphangiogenesis and metastasis via upregulation of VEGF-C in mouse models of breast cancer. J Clin Invest 122: 1895-1906, 2012.

30. Yu Y, Davicioni E, Triche TJ and Merlino G: The homeoprotein six1 transcriptionally activates multiple protumorigenic genes but requires ezrin to promote metastasis. Cancer Res 66 1982-1989, 2006

31. Wu K, Li Z, Cai S, Tian L, Chen K, Wang J, Hu J, Sun Y, Li X, Ertel A and Pestell RG: EYA1 phosphatase function is essential to drive breast cancer cell proliferation through cyclin D1. Cancer Res 73: 4488-4499, 2013

32. Christensen KL, Brennan JD, Aldridge CS and Ford HL: Cell cycle regulation of the human Six 1 homeoprotein is mediated by APC(Cdh1). Oncogene 26: 3406-3414, 2007.

33. Ford HL, Landesman-Bollag E, Dacwag CS, Stukenberg PT, Pardee AB and Seldin DC: Cell cycle-regulated phosphorylation of the human SIX1 homeodomain protein. J Biol Chem 275: 22245-22254, 2000.

34. Iwanaga R, Wang CA, Micalizzi DS, Harrell JC, Jedlicka P, Sartorius CA, Kabos P, Farabaugh SM, Bradford AP and Ford HL: Expression of Six1 in luminal breast cancers predicts poor prognosis and promotes increases in tumor initiating cells by activation of extracellular signal-regulated kinase and transforming growth factor-beta signaling pathways. Breast Cancer Res 14: R100, 2012. 